\section{A tale of two trials: selectively replicating herpesviruses for brain tumors}

Cancer gene therapy, the genetic modification of cells for therapeutic benefit against cancer, holds great promise. Encouraging antitumoral efficacy and safety has been demonstrated in preclinical animal models using varied approaches, including tumor suppressor gene replacement, prodrug-activating enzyme expression and immunomodulatory strategies. However, clinical trial results have been disappointing to date. Although serious treatment-related toxicities have not been demonstrated in general, antitumoral efficacy has been minimal or nonexistent. The reasons for this are certainly varied, but clearly two major limitations have been the inability to achieve: (1) high-level gene expression; (2) in sufficient numbers of target cells to result in clinical benefit. These limitations need to be improved upon if cancer gene therapy is ever to fulfil its promise.

One novel and potentially fruitful approach to achieving higher-level gene expression and more widespread infection within tumors is the use of replication-selective agents. ${ }^{1}$ Alternatively referred to as 'oncolytic' agents or as 'cancer biotherapy', this approach capitalizes on the fact that microbial agents such as viruses and bacteria can replicate within human tissues to levels that are many logs higher than the input 'dose'. Replication leads to high-level gene expression from both the infecting agent and its progeny and in many cases, to death of the infected cell. In addition to direct lysis at the conclusion of the replicative cycle, these agents can kill cells through expression of toxic proteins, induction of both inflammatory cytokines and $\mathrm{T}$ cell-mediated immunity, and enhancement of cellular sensitivity to their effects. Tumor selectivity appears to be an inherent property of viruses such as autonomous parvoviruses and reovirus, ${ }^{2}$ whereas tumor selectivity can be genetically engineered in the cases of herpesvirus, ${ }^{3}$ adenovirus ${ }^{4}$ and Salmonella typhimurium. ${ }^{5}$

Although preclinical data reported with these agents have been encouraging, many critical questions have awaited results from clinical trials. These microbial agents have complex biologies, including species-specific interactions with host cell machinery and/or immune response effectors. Antitumoral efficacy and safety studies are frequently performed in different species. Therefore, data from cancer patients have been eagerly awaited. The first clinical trial results reported with a replication-selective adenovirus were those from phase I and II trials of dl1520 (ONYX-015; now known as CI-1042, Parke-Davis Pharmaceutical Division of Warner-
Lambert). ${ }^{6}$ Following direct injection of both tumor and normal peritumoral tissues, viral replication and cytopathic effects were limited to tumor tissues only. The treatment has also been well tolerated following intravascular administration. However, clinical data from other replication-selective agents were lacking.

In this issue of Gene Therapy, the eagerly awaited results of two trials with replication-selective herpes simplex viruses (HSV-1 mutants) are reported. The Scottish group evaluated the ICP34.5 null mutant 1716, ${ }^{7}$ whereas the group from the USA tested G207,, a mutant with deletions in ICP34.5 and ribonucleotide reductase genes. ICP34.5 was deleted because of its role in neuropathogenesis, while the ribonucleotide reductase mutation was designed to limit replication to proliferating cells (neurons in the brain are quiescent and would therefore be protected). Both groups performed phase I dose-escalation safety trials in patients with incurable malignant brain tumors, primarily glioblastoma multiforme. Although the study populations and injection techniques were similar, patients on the Scottish trial $(n=9)$ received significantly lower doses of virus $\left(10^{3}-10^{5}\right.$ plaque-forming units (p.f.u.)) than patients on the US trial $(n=21$; $10^{6}-3 \times 10^{9}$ p.f.u.); the highest dose levels therefore differed by 30000 -fold. Both sets of investigators, their respective regulatory agencies and gene therapy oversight committees are all to be highly commended for their efforts on behalf of these extremely challenging and complex clinical trials with two such novel therapies in terminally ill patients.

The safety results reported from both trials are reassuring. Up to $3 \times 10^{9}$ p.f.u. (approximately $3 \times 10^{11}$ particles) of mutant HSV could be injected directly into brain tumors without significant toxicity attributed to treatment. Although uncommon or relatively mild adverse effects could easily be missed in small trials such as these, this apparent degree of safety is remarkable given the immunosuppressive medications taken by all study patients. Although these trials were not designed to evaluate efficacy, both groups used detailed volumetric measurements to assess antitumoral effects. No consistent changes could be demonstrated on post-injection MRI scans, but anecdotal cases of tumor shrinkage or prolonged progression-free intervals were encouraging. Further clinical evaluation of these agents is clearly indicated.

However, both of these trials are also disappointing because of the questions they leave unanswered. Neither study identified a maximally tolerated dose nor the doselimiting toxicity of the treatment, although the G207 trial appears to have treated patients at the highest available 
dose. Clinical development of novel, and potentially nontoxic, agents such as these may require novel phase I endpoints; nevertheless, further dose escalation appears to be indicated in future trials, if feasible. In addition, no reliable data on viral replication could be obtained from tumor tissue due to both ethical and technical considerations. While repeat post-treatment biopsies have routinely been performed to assess viral replication in patients with accessible tumors (such as in the head and neck region), this is not feasible in brain tumor patients. Access to tumor tissue occurred sporadically at either surgery or at autopsy. We therefore cannot be sure whether the safety and lack of viral shedding into the saliva and blood is indicative of a highly controlled infection or a paucity of replication. Fortunately, each of these unanswered questions can be answered in future trials that enrol patients with more easily accessible tumors; HSV can replicate in a wide variety of epithelial carcinomas.

The future of the biotherapy treatment platform is bright. Clinical trial results with adenoviral mutants and now herpesviruses document that genetically engineered viruses can be well tolerated and associated with biological activity. Once safety has been established, the focus of investigators can shift to improving the efficacy of these treatments. Replication-selective agents can be combined with standard cancer treatments, ${ }^{9,10}$ (eg chemotherapy, radiotherapy and surgery) and can be 'armed' with therapeutic genes to boost potency. ${ }^{11}$ Regardless of the treatment regimen, detailed biological endpoints will be a critical focus of future trials. Novel approaches to noninvasive imaging will be an important complement to traditional biopsy and blood analyses. Only then can the true promise of this remarkable approach be realized.

DH Kirn

Imperial Cancer Research Fund Viral and Genetic Therapy Programme ICSTM at Hammersmith Hospital

Du Cane Road

London W12 ONN, UK

\section{References}

1 Kirn D. Replication-selective micro-organisms: fighting cancer with targeted germ warfare. J Clin Invest 2000; 105: 836-838.

2 Coffey M, Strong J, Forsyth P, Lee P. Reovirus therapy of tumors with activated ras pathway. Science 1998; 282: 1332-1334.

3 Mineta $\mathrm{T}$ et al. Attenuated multi-mutated herpes simplex virus1 for the treatment of malignant gliomas. Nature Med 1995; 1: 938-943.

4 Heise C et al. ONYX-015, an E1B gene-attenuated adenovirus, causes tumor-specific cytolysis and antitumoral efficacy that can be augmented by standard chemotherapeutic agents (see comments). Nature Med 1997; 3: 639-645.

5 Pawelek J, Low K, Bermudes D. Tumor-targeted Salmonella as a novel anticancer vector. Cancer Res 1997; 57: 4537-4544.

6 Kirn D et al. A phase II trial of intratumoral injection with an E1B-deleted adenovirus, ONYX-015, in patients with recurrent, refractory head and neck cancer. Proc Am Soc Clin Oncol 1998; 17: 391a.

7 Rampling $\mathrm{R}$ et al. Toxicity evaluation of replication-competent herpes simplex virus (ICP 34.5 null mutant 1716) in patients with recurrent malignant glioma. Gene Therapy 2000; 7: 859-866.

8 Markert JM et al. Conditionally replicating herpes simplex virus mutant, G207 for the treatment of malignant glioma: results of a phase I trial. Gene Therapy 2000; 7: 867-874.

9 Rogulski $\mathrm{K}$ et al. In vivo antitumor activity of ONYX-015 is influenced by p53 status and is augmented by radiotherapy. Cancer Res 2000; 60: 1193-1196.

10 Kirn DH et al. A phase II trial of ONYX-015, a selectively-replicating adenovirus, in combination with cisplatin and 5-fluorouracil in patients with recurrent head and neck cancer. Proc Am Soc Clin Oncol 1999; 18: 1505.

11 Freytag SO et al. A novel three-pronged approach to kill cancer cells selectively: concomitant viral, double suicide gene, and radiotherapy (see comments). Hum Gene Ther 1998; 9: 1323-1333. 